\title{
The efficacy and safety of phototherapy in geriatric patients: a retrospective study*
}

\author{
Isil Bulur ${ }^{1}$ \\ Ayse Esra Aksu² \\ Zeynep Nurhan Saracoglu ${ }^{1}$ \\ DOI: http:/ / dx.doi.org/10.1590/abd1806-4841.20185468
}

Hilal Kaya Erdogan ${ }^{1}$

Tekden Karapınar ${ }^{1}$

\begin{abstract}
BACKGROUND: While phototherapy is a well-established treatment for many dermatoses, data from the literature regarding its use in elderly patients are quite limited.

OвJECTIVE: In this study, we aimed to determine the phototherapy indications in geriatric patients and to evaluate the effectiveness and reliability of phototherapy in this group.

MetHODS: This study included 95 patients of 65 years of age and older who were treated in our phototherapy unit between 2006 and 2015. The data for this study were collected retrospectively from patient follow-up forms in the phototherapy unit. REsults: Phototherapy was administered to 28 (29.5\%) patients for mycosis fungoides, 25 (26.3\%) patients foplaque type psoriasis, $12(12.6 \%)$ patients for palmoplantar psoriasis, 12 (12.6\%) patients for generalized pruritus, and 18 (19\%) for other dermatoses. Of the patients, $64.2 \%$ had received a narrowband UVB (NB-UVB), $21.1 \%$ oral psoralen UVA (PUVA), and $14.7 \%$ local PUVA treatment. A complete response was achieved in $76.9-85.7 \%$ of the mycosis fungoides and in $73.71-100 \%$ of the psoriasis vulgaris patients treated with NB-UVB and PUVA, respectively. All the patients with generalized pruritus were treated with NB-UVB, and $80 \%$ of these patients achieved significant improvement. The erythema rate was found to be $0.43 \%$ per session for NB-UVB treatment and $0.46 \%$ per session for PUVA treatment as a side effect.

StUDY LIMITATIONS: The limitations of our study are that it was retrospective and the remission durations of the patients are not known. Conclusion: This study showed that phototherapy is effective and reliable in the elderly population with proper dose increases and close follow-up.
\end{abstract}

Keywords: Health of the elderly; Phototherapy; Skin diseases

\section{INTRODUCTION}

Nowadays, there is an increase in the geriatric population in both developed and developing countries. According to 2014 data, $8.3 \%$ of the world population is older than 65 years of age, and this ratio is $8 \%$ in Turkey. ${ }^{1}$ When observed demographically, this change causes important health problems and makes it mandatory to arrange healthcare services accordingly. In addition to systemic disorders, dermatologic diseases are also important in terms of affecting the quality of life in this age group. ${ }^{2}$ Being aware of the metabolic and physiological changes with age and considering the accompanying comorbidities and drug use in these patients are important in terms of preventing iatrogenic complications when selecting the treatment method. ${ }^{3}$ Although topical treatments can also be used for some dermatologic disorders in order to prevent the side effects of systemic treatments, phototherapy becomes an important treatment choice in cases where topical treatments are ineffective. Phototherapy is a treatment method with proven effectiveness and reliability in the adult age group, but the data in the literature regarding its use in the elderly population are inadequate.

The present study aimed to determine the phototherapy indications in patients of 65 years of age and older, who were followed up in our phototherapy unit between 2006 and 2015, and to evaluate the efficacy and safety of phototherapy in commonly seen dermatoses in this age group.

Received on 04.12.2015.

Approved by the Advisory Board and accepted for publication on 09.08.2016.

* Work performed at the Department of Dermatology, Eskişehir Osmangazi University, Faculty of Medicine, Eskişehir, Turkey.

Financial support: None

Conflict of interest: None.

1 Department of Dermatology, Faculty of Medicine, Eskisehir Osmangazi University, Eskişehir, Turkey.

2 Deparrment of Dermatology, Istanbul Training and Research Hospital, Istanbul, Turkey.

MAILING ADDRESS:

Isil Bulur

E-mail: isilbulur@yahoo.com

(C2018 by Anais Brasileiros de Dermatologia 


\section{METHODS}

This study included 95 patients of 65 years of age and older, who were followed up in our phototherapy unit between 2006 and 2015. The phototherapy data were evaluated retrospectively from the follow-up forms of the phototherapy unit.

\section{Phototherapy Protocol}

Narrowband UVB (NB-UVB) treatment and oral psoralen UVA (PUVA) were administered with the Daavlin Spectra 305/350 model UV device to the patients included in the study. All treatment protocols were started at three times a week. NB-UVB treatment was started with $70 \%$ of the minimal erythema dose (MED), and PUVA treatment was started with $70 \%$ of the minimal phototoxic dose (MPD). The subsequent dosage increased by $10-20 \%$ of the previous dose at each session, according to the clinical situation of the patient for NB-UVB. 8-methoxypsoralen was administered at a dose of $0.6 \mathrm{mg} / \mathrm{kg}$ for PUVA, and treatment was continued with $0.5 \mathrm{j}$ increases weekly. Treatment was continued with $10 \%$ increases in the case of minimal erythema presence during the treatment with NB-UVB. The dose was not increased in the case of continuous moderate erythema. The treatment was interrupted until symptoms regressed in the cases of severe erythema, edema and bullae development. After symptoms regressed, treatment was continued with $50 \%$ of the last dose, and dose increases were determined as $10 \%$. Treatment was continued without an increase in PUVA treatment in the case of the presence of minimal-mild erythema. The treatment was interrupted until symptoms regressed in the cases of severe erythema, edema, and bullae development. After symptoms regressed, treatment was started with $50 \%$ of the last dose and continued with $10 \%$ increases.

Patients who used only topical corticosteroids, moisturizers, or antihistamines were included in the study. Patients who were treated with phototherapy for less than 8 sessions (due to their own request) were excluded from the evaluation of the relationship between the disorder and the phototherapy dose, effects, and side effects.

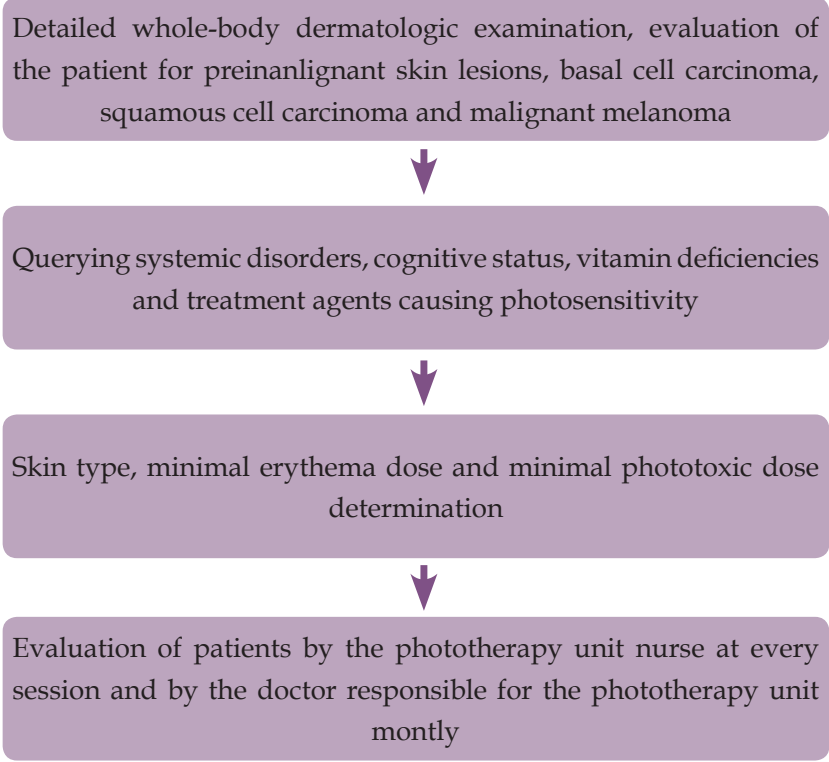

FIGURE 1: Follow-up steps for elderly patients receiving phototherapy
The steps followed up before and during the phototherapy treatment in individuals of 65 years of age and older in our clinic are presented in figure 1 . Besides liver and renal function tests, complete blood cell counts were performed monthly for the evaluation of 8-MOP side effects in patients treated with PUVA.

\section{Evaluation of the treatment response and termination of} treatment.

Early stage (Stage 1A, 1B, 2A) mycosis fungoides patients were included in the study. A $90-100 \%$ disappearance of the lesions with histopathological support of treatment response was accepted as a complete response in mycosis fungoides patients. PASI 75 and over was evaluated as a response for plaque type psoriasis vulgaris. Improvement of more than $75 \%$ in the initial lesion for the other dermatoses and complete improvements in the symptom of itching according to the feedback of the patient were accepted as recovery. The treatment was continued until total remission was obtained or no additional improvement was seen in the following sessions.

\section{Statistical Analysis}

The analysis of the data was performed using the SPSS for Windows 11.5 software program. Descriptive statistics for continuous variables were shown as mean \pm standard deviation or median (minimum-maximum), and categorical variables were shown as the number of cases (\%). Fisher's exact test was used for the comparison of two proportions. The chi-squared test was used in the analysis of quantitative data. Results were accepted as statistically significant for $\mathrm{p}<0.05$.

\section{RESULTS}

This study included 95 patients (43 female, 52 male) with a mean age of $71.6 \pm 5.4$ years. The skin type was type 2 in $57(60.0 \%)$ patients and type 3 in 38 (40\%) patients. Mycosis fungoides (29.5\%) was the most common indication for phototherapy, followed by plaque type psoriasis (26.3\%), palmoplantar psoriasis (12.6\%), and generalized pruritus (12.6\%) (Table 1). Other disorders treated with phototherapy were lichen planus, contact dermatitis, atopic dermatitis, morphea,

TABLE 1: Distribution of patients according to phototherapy recommendation and phototherapy type

Total Number DUVB PUVA Local

of Patients $n \quad n \quad$ PUVA

\begin{tabular}{lcccc} 
& n (\%) & & & n \\
\hline Mycosis fungoides & $28(29.5)$ & 14 & 14 & \\
\hline Psoriasis & $25(26.3)$ & 22 & 3 & \\
Generalized pruritus & $12(12.6)$ & 12 & & \\
Palmoplantar psoriasis & $12(12.6)$ & & & 12 \\
\hline Lichen planus & $8(8.4)$ & 8 & & \\
Contact dermatitis & $4(4.2)$ & & & 4 \\
Atopic dermatitis & $2(2.1)$ & 2 & & \\
Morphea & $1(1.1)$ & & 1 & \\
Keratoderma & $1(1.1)$ & & & 1 \\
Pigmented purpuric & $1(1.1)$ & & 1 & \\
dermatosis & & & &
\end{tabular}


keratoderma, pigmented purpuric dermatitis, and generalized porokeratosis (Table 1). Of the patients, 61 (64.2\%) had received NB-UVB, 20 (21.1\%) PUVA, and 14 (14.7\%) local PUVA treatment (Graph 1).

\section{Mycosis Fungoides $(n=28)$}

Mycosis fungoides was the most common at $29.5 \%$ among the disorders with a phototherapy indication. Fourteen $(50 \%)$ patients were treated with NB-UVB, and $14(50 \%)$ patients were treated with PUVA (Table 1). One patient treated with PUVA discontinued the treatment before completing 8 sessions. The median age was 75 (65-82) years for the 14 patients who received NB-UVB treatment and 68 (65-84) years for the 13 patients who received PUVA treatment. The median number of sessions was 55 (10-148), with a median cumulative dose of $59.3(5.2-240) \mathrm{j} / \mathrm{cm}^{2}$, for NB-UVB treatment, while the median number of sessions was 39 (10-146), with a median cumulative dose of 253.0 (35-1242) j/ $\mathrm{cm}^{2}$, for PUVA treatment. No statistically significant difference was found between NB-UVB and PUVA treatments in terms of the number of sessions $(p=0.616)$. Erythema was found as a side effect in $3(21.4 \%)$ patients treated with NB-UVB and $4(28.6 \%)$ patients treated with PUVA. A complete response was obtained with treatment in $85.7 \%$ of the patients treated with NB-UVB and in $76.9 \%$ of the patients treated with PUVA. No statistically significant difference was found between those who were treated with

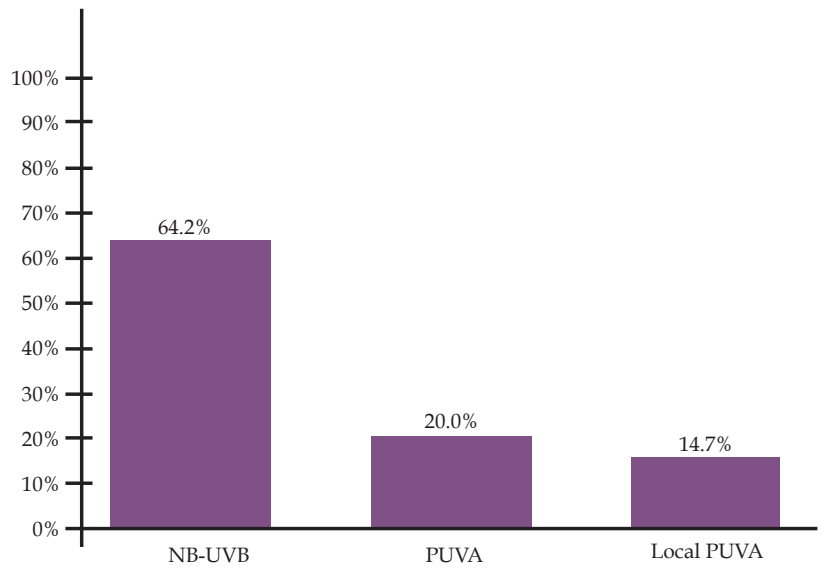

GRAPH 1: Distribution of patients based on therapy received
NB-UVB and PUVA in terms of the rate of benefiting from the treatment $(p=0.367)$. Parameters regarding NB-UVB and PUVA treatment in mycosis fungoides patients are summarized in table 2.

\section{Psoriasis $(n=25)$}

Plaque type psoriasis was present in $26.3 \%$ of the patients included in the study (Table 2). NB-UVB treatment was used in $22(88 \%)$ patients and PUVA treatment in $3(12 \%)$ patients. Three patients treated with NB-UVB discontinued the treatment before completing 8 sessions. The median age was 71 (65-80) for NB-UVB and 68 (66-77) for PUVA. The median number of sessions was 42 (14-170), with a median cumulative dose of $66.8(4.9-198.9) \mathrm{j} / \mathrm{cm}^{2}$, for NB-UVB treatment, while the median number of sessions was 25 (19-69), with a median cumulative dose of 219.8 (90-873) j/ $\mathrm{cm}^{2}$, for PUVA treatment. PASI 75 response was achieved in $73.7 \%$ of the patients treated with NB-UVB and in all of the patients treated with PUVA. Erythema was found as a side effect in 7 (36.8\%) patients who received NB-UVB treatment and in 1 (33.3\%) patient who received PUVA treatment. Parameters regarding NB-UVB and PUVA treatment in psoriasis patients are summarized in table 3.

\section{Generalized Pruritus $(n=12)$}

Generalized pruritus was present in $12.6 \%$ of the patients included in this study, in which all of the patients received NB-UVB treatment. Two patients who started phototherapy discontinued the treatment before completing 8 sessions. The median age of the patients was 69 (65-81) years. The median number of sessions was 33.5 (12-108), with a median cumulative dose of $50.5(4.5-205) \mathrm{j} / \mathrm{cm}^{2}$. The number of patients who benefited from NB-UVB treatment was 8 (80\%). Erythema was observed in $2(20 \%)$ patients as a side effect. Parameters regarding NB-UVB treatment in generalized pruritus patients are summarized in table 4 .

\section{Lichen Planus $(n=8)$}

All the patients with lichen planus received NB-UVB treatment, and a response was achieved in $37.5 \%$ of the patients, with a median cumulative dose of $21.4(4.9-99.7) \mathrm{j} / \mathrm{cm}^{2}$ and a median number of 19 (9-60) sessions.

TABLE 2: Features of Patients with Mycosis Fungoides

\begin{tabular}{|c|c|c|c|c|}
\hline & \multicolumn{4}{|c|}{ Mycosis Fungoides } \\
\hline & \multicolumn{2}{|c|}{ DUVB $(n=14)$} & \multicolumn{2}{|c|}{ PUVA(n=13) } \\
\hline & Mean \pm SD & Median (range) & Mean \pm SD & Median (range) \\
\hline Age (years) & $74.1 \pm 6.5$ & $75(65-82)$ & $70.5 \pm 6.5$ & $68.0(65-84)$ \\
\hline Number of Sessions & $55.4 \pm 35.4$ & $55.0(10-148)$ & $52.7 \pm 41.1$ & $39(10-146)$ \\
\hline MED/MFD dose $\left(\mathrm{mj} / \mathrm{cm}^{2}-\mathrm{j} / \mathrm{cm}^{2}\right)$ & $540.5 \pm 153.2$ & $575(245-800)$ & $3.3 \pm 1.4$ & $3.5(1-5)$ \\
\hline First dose $\left(\mathrm{mj} / \mathrm{cm}^{2}-\mathrm{j} / \mathrm{cm}^{2}\right)$ & $335.8 \pm 78.8$ & $332(166-420)$ & $2.0 \pm 0.9$ & $2.3(1-3)$ \\
\hline Maximum Dose $\left(\mathrm{mj} / \mathrm{cm}^{2}-\mathrm{j} / \mathrm{cm}^{2}\right)$ & $1400 \pm 588.4$ & $1336(492-2100)$ & $6.0 \pm 2.6$ & $6.0(3-11)$ \\
\hline Cumulative Dose $\left(\mathrm{j} / \mathrm{cm}^{2}\right)$ & $70.3 \pm 60.4$ & $59.3(5.2-240)$ & $363.2 \pm 357.7$ & $253.0(35-1242)$ \\
\hline Side Effect & \multicolumn{2}{|c|}{$3(21.4 \%)$} & \multicolumn{2}{|c|}{$4(30.8 \%)$} \\
\hline Treatment Response & \multicolumn{2}{|c|}{$12(85.7 \%)$} & \multicolumn{2}{|c|}{$10(76.9 \%)$} \\
\hline
\end{tabular}

MED: minimal erythema dose, MFD: minimal phototoxic dose 


\section{Other Dermatoses}

Two patients diagnosed with atopic dermatitis were treated with NB-UVB. The treatment response was observed in 1 patient who underwent 43 sessions and received a $64.8 \mathrm{j} / \mathrm{cm}^{2}$ cumulative dose, while in the other patients underwent 13 sessions and received an $8.7 \mathrm{j} / \mathrm{cm}^{2}$ cumulative dose. One 67 -year-old female patient, with pigmented purpuric dermatoses, was treated with PUVA, and a complete response was obtained in 40 sessions with a cumulative dose of $181 \mathrm{j} / \mathrm{cm}^{2}$. In addition, one 65-year-old male patient, with generalized porokeratosis, received PUVA treatment and a complete response was achieved upon the $42^{\text {nd }}$ session.

\section{Side Effects}

The photosensitive drugs that the patients most often used are summarized in table 5 in their order of frequency. No acute adverse effect required the discontinuation of therapy. Erythema was observed in $35 \%$ of the patients treated with NB-UVB and in $33 \%$ of those treated with PUVA. The incidence of erythema was found to be $0.43 \%$ per session in NB-UVB treatment and $0.46 \%$ per session in PUVA treatment. The erythema incidence showed no statistically significant difference between NB-UVB and PUVA treatments $(p=0.111)$. No gastric side effects requiring the discontinuation of the treatment was observed in any of the patients who received PUVA treatment.

\section{DISCUSSION}

This study demonstrated that phototherapy is effective and reliable in the elderly population. Studies evaluating skin disorders and quality of life in the elderly population are available in the literature, but, to date, only one study has evaluated the use of phototherapy in this population. ${ }^{4}$

Powell et al. reported that psoriasis was the most common skin disorder, with $51 \%$ of 37 elderly patients treated with phototherapy, while in the present study mycosis fungoides $(29.5 \%)$ was the disorder treated most frequently with phototherapy in the elderly. ${ }^{4}$ Mycosis fungoides is mainly observed after 55-60 years of age, and phototherapy is included among the first-step treatments for early stage mycosis fungoides (stage 1A, 1B, 2A). ${ }^{5-6}$ Therefore, this result is not unexpected.
The first option in phototherapy for the mycosis fungoides patients in our clinic is PUVA treatment. However, we prefer to apply NB-UVB treatment in geriatric patients with systemic disorders and multiple drug use due to the psoralen's gastric side effects and liver toxicity. The clinical response rate varies between $42 \%$ and $86 \%$, with PUVA administered three times a week in early stage mycosis fungoides patients. ${ }^{7}$ In this study, a complete response rate in mycosis fungoides was obtained in $76.9 \%$ of the patients treated with PUVA, with a median of 39 sessions and a median cumulative dose of $253.0 \mathrm{j} / \mathrm{cm}^{2}$. Ponte et al. reported that clinical response was achieved in $62.1 \%$ of their early stage mycosis fungoides patients, with a mean number of 31 sessions and a cumulative dose of 123.8 $\mathrm{j} / \mathrm{cm}^{2}{ }^{8}$ On the other hand, they pointed out that retinoid treatment was given in addition to phototherapy in $53.7 \%$, corticosteroid treatment in $3.2 \%$, and methotrexate treatment in $2.1 \%$ of the patients included in their study. ${ }^{9}$ In another study, $64 \%$ of the patients with mycosis fungoides treated with PUVA achieved a complete response, with an average of 19.5 (7-50) sessions. ${ }^{9}$

The response rate with NB-UVB in mycosis fungoides cases varies between $54 \%$ and $91 \%$ in other studies, and the number of sessions for full recovery with NB-UVB is reported to be between 19 and $36 .{ }^{7}$ In our study, a complete response was observed in $85.7 \%$ of the patients with mycosis fungoides who were treated with NBUVB, with a median number of 55 sessions and a cumulative dose of

\begin{tabular}{|lcc|}
\hline \multicolumn{3}{|c|}{ TABLE 4: Features of the patients with pruritus } \\
\cline { 2 - 3 } & \multicolumn{3}{c|}{ DUVB (n=10) } \\
\hline Mge (years) & Mean \pm SD & Median (range) \\
\hline Number of Sessions & $70.9 \pm 5.3$ & $69(65-81)$ \\
\hline Med dose $\left(\mathrm{mj} / \mathrm{cm}^{2}\right)$ & $54.7 \pm 29.3$ & $33.5(12-108)$ \\
\hline First dose $\left(\mathrm{mj} / \mathrm{cm}^{2}\right)$ & $360.8 \pm 77.3$ & $375(243-480)$ \\
\hline Maximum Dose $\left(\mathrm{mj} / \mathrm{cm}^{2}\right)$ & $1516 \pm 454.9$ & $1541(751-2148)$ \\
\hline Cumulative Dose $\left(\mathrm{j} / \mathrm{cm}^{2}\right)$ & $63.2 \pm 56.6$ & $50.5(4.5-205)$ \\
\hline Side Effect & \multicolumn{2}{|c|}{$2(20.0 \%)$} \\
\hline Treatment response & \multicolumn{2}{|c|}{$8(80.0 \%)$} \\
\hline
\end{tabular}

MED: minimal erythema dose

\begin{tabular}{|c|c|c|c|c|}
\hline & \multicolumn{4}{|c|}{ Psoriasis Vulgaris } \\
\hline & \multicolumn{2}{|c|}{ DUVB $(n=19)$} & \multicolumn{2}{|c|}{ PUVA $(n=3)$} \\
\hline & Mean \pm SD & Median (range) & Mean $\pm S D$ & Median (range) \\
\hline Age (years) & $71.3 \pm 5.0$ & $71(65-80)$ & $70.3 \pm 5.9$ & $68(66-77)$ \\
\hline Number of Sessions & $55.1 \pm 38.7$ & $42.0(14-170)$ & $36.0 \pm 24.4$ & $25(19-69)$ \\
\hline Med dose $\left(\mathrm{mj} / \mathrm{cm}^{2}-\mathrm{j} / \mathrm{cm}^{2}\right)$ & $504.0 \pm 135.7$ & $486(250-800)$ & $2.7 \pm 1.4$ & $3.5(1-4)$ \\
\hline First dose $\left(\mathrm{mj} / \mathrm{cm}^{2}-\mathrm{j} / \mathrm{cm}^{2}\right)$ & $285.6 \pm 79.2$ & $300(150-480)$ & $1.5 \pm 0.9$ & $1(1-3)$ \\
\hline Maximum Dose $\left(\mathrm{mj} / \mathrm{cm}^{2}-\mathrm{j} / \mathrm{cm}^{2}\right)$ & $1466.8 \pm 644.7$ & $1368(350-2458)$ & $12.23 \pm 7.5$ & $15(4-18)$ \\
\hline Cumulative Dose $\left(\mathrm{j} / \mathrm{cm}^{2}\right)$ & $70.0 \pm 50.8$ & $66.8(4.9 \pm 198.9)$ & $394.3 \pm 419.6$ & $219.8(90-873)$ \\
\hline Side Effect & \multicolumn{2}{|c|}{$7(36.8 \%)$} & \multicolumn{2}{|c|}{$1(33.3 \%)$} \\
\hline Treatment Response & \multicolumn{2}{|c|}{$14(73.7 \%)$} & \multicolumn{2}{|c|}{$3(100 \%)$} \\
\hline
\end{tabular}

MED: minimal erythema dose, MFD: minimal phototoxic dose 


\begin{tabular}{|c|c|c|}
\hline \multicolumn{2}{|c|}{ Drugs } & The Number of the patients $(\mathrm{n} / \%)$ \\
\hline Antihistamines & Hydroxyzine & $43(45.3)$ \\
\hline Non-steroidal anti-inflammatory drugs & Diclofenac, ibuprofen, ketoprofen, naproxen, & $37(38.9)$ \\
\hline Cardiovascular drugs & Atenolol, nifedipine, captopril & $12(12.6)$ \\
\hline Diuretics & Amiloride, furosemide, hydrochlorothiazide & $8(0.1)$ \\
\hline Antidiabetics & Sulfonylurea & $6(0.1)$ \\
\hline
\end{tabular}

$59.3 \mathrm{j} / \mathrm{cm}^{2}$. Boztepe et al. reported that a full response was obtained in $78.6 \%$ of their patients, with a cumulative dose of $108.8 \pm 40.8 \mathrm{j} \mathrm{cm}{ }^{2}$ and a median number of sessions of $22.7 \pm 5.4$ for stage $1 \mathrm{~A}-1 \mathrm{~B}$ and $30.2 \pm 12.8$ for stage $2 \mathrm{~A}$ in patients with NB-UVB in the 14 mycosis fungoides patients evaluated by their research team. ${ }^{10}$

Systemic treatment administration is quite difficult in moderate and severe psoriasis in the elderly population due to the accompanying comorbidities and multiple drug use. On the other hand, phototherapy is a reliable treatment method with fewer side effects in this patient group. However, the evaluation of previous phototherapy doses and immunosuppressive drugs received by the patients is quite important before the phototherapy decision is made in psoriasis. Our study revealed a PASI 75 response in $73.7 \%$ of the 19 psoriasis vulgaris patients treated with NB-UVB, with a median number of 42.0 sessions and a median cumulative dose of $66.8 \mathrm{j} / \mathrm{cm}^{2}$. All patients $(\mathrm{n}=3)$ treated with PUVA achieved a PASI 75 response with a median number of 25 sessions and a median cumulative dose of $219.8 \mathrm{j} / \mathrm{cm}^{2}$. Yones et al. observed full recovery in $84 \%$ of their chronic plaque type psoriasis patients who received PUVA, with a median number of 17 sessions, and in $65 \%$ of those who received NB-UVB, with a median number of 28.5 sessions. ${ }^{11}$ Markham et al. reported that they observed an improvement in psoriasis patients in 25.5 (18-32.5) sessions, with NB-UVB and in 19 (14.6-25.0) sessions with PUVA..$^{12}$

Pruritus is a common dermatologic problem in the elderly population with a prevalence of $29 \%$ and is usually idiopathic in this age group. ${ }^{13}$ There is a scarcity of publications on phototherapy in generalized pruritus. ${ }^{14-16}$ Seçkin et al. reported obtaining relief in $68 \%$ of the 25 idiopathic pruritus cases, with a mean age of 51.7 (range 2591) years, with a mean number of 22 sessions and cumulative dose of $20801 \mathrm{mj} / \mathrm{cm}^{2} .{ }^{14}$ In our study, NB-UVB treatment was administered to all of the patients with idiopathic generalized pruritus, in which complete relief was observed in $80 \%$ of the patients, with a median number of 33.5 sessions and a median cumulative dose of $50.5 \mathrm{j} / \mathrm{cm}^{2}$.

All of the patients with lichen planus were treated with NB$\mathrm{UVB}$, and a significant improvement was obtained from the treatment in $37.5 \%$ of the patients, with a cumulative median dose of $21.4 \mathrm{j} / \mathrm{cm}^{2}$ in a median number of 19 sessions. Gamil et al. reported that $68.8 \%$ of their patients who received NB-UVB at a cumulative dose of $84.5 \pm 31.6$ achieved a complete response, while $70 \%$ of the patients who received NB-UVB in another study showed significant improvement in a mean number of 10.9 weeks. ${ }^{17,18}$ Saricaoğlu et al. observed a complete response in $50 \%$ of the patients treated with NB-UVB in 30 sessions. $^{19}$

This study shares similar results with other studies. However, the number of sessions in our study is higher than in the oth- er studies in the literature. The reason for this difference may be explained by the fact that that our patients were generally treated with a low increment regimen. We could not discuss the impact of phototherapy for other dermatoses in elderly patients, due to low number of the patients.

Polypharmacy in elderly patients is important due to their phototoxic and photoallergic side effects, in addition to their hepatic and renal side effects, during phototherapy treatment. The patients should be advised not to use additional systemic and topical drugs without asking their doctors. Moreover, we recommend that the clinician should bear in mind that commonly used non-steroidal anti-inflammatory drugs and antihistamines in this population can cause phototoxic and photoallergic reactions. Although we did not observe severe acute adverse effects that would require the interruption of treatment in any of our patients in this study, erythema was observed in $35 \%$ of the patients treated with NB-UVB and in $33 \%$ of the patients treated with PUVA. Markham et al. reported that first degree erythema was observed in $75 \%$ of the mycosis fungoides patients treated with NB-UVB and in $80 \%$ of the patients treated with PUVA. ${ }^{12}$ Martin et al. reported that an acute adverse event was observed in $0.6 \%$ of NB-UVB treatments and $1.3 \%$ of systemic PUVA treatments in their study where they evaluated the acute side effects of these two methods. ${ }^{20}$ In our study, the incidence of erythema per treatment was $0.43 \%$ for NB-UVB treatment and $0.46 \%$ for PUVA treatment. No side effect, except slightly elevated liver enzymes secondary to psoralen, which would require the interruption of treatment, was found in any of the patients treated with PUVA. No difference was observed between the two treatment groups in terms of side effects. However, the low number of patients and the fact that PUVA treatment was not used in the risky patient group may have caused this result. Late side effects of phototherapy such as photoaging and photocarcinogenicity, were not evaluated in our patients.

The limitations of our study are that it was retrospective and that the remission durations of the patients are unknown. However, this study is valuable as the number of patients is high, and there are no other studies in the literature that evaluate the number of phototherapy sessions, dose, effectiveness, and side effects of phototherapy together in the elderly population.

\section{CONCLUSION}

In conclusion, the incidence of the response rate and adverse effects in our study is consistent with results from other studies in the literature, and our data shows that phototherapy is effective and reliable with proper dose increases and close follow-up in geriatric patients. $\square$ 


\section{REFERENCES}

1. Tuik.gov.tr [Internet]. Elderly with statistics. Turkey: Turkish Statistical Institute 2014. [cited 2015 Nov 10]. Available from: http: //. www.tuik.gov.tr/PdfGetir do? id $=18620$

2. Shah $M$, Coates $M$. An assessment of the quality of life in older patients with skin disease. Br J Dermatol. 2006;154:150-3.

3. Endo JO, Wong JW, Norman RA, Chang AL. Geriatric dermatology: Part I. Geriatric pharmacology for the dermatologist. J Am Acad Dermatol. 2013;68:521.e1-10.

4. Powell JB, Gach JE. Phototherapy in the elderly. Clin Exp Dermatol. 2015 Aug;40:605-10.

5. Jawed SI, Myskowski PL, Horwitz S, Moskowitz A, Querfeld C. Primary cutaneous T-cell lymphoma (mycosis fungoides and Sézary syndrome): part I. Diagnosis: clinical and histopathologic features and new molecular and biologic markers. J Am Acad Dermatol. 2014;70:205.e1-16.

6. Jawed SI, Myskowski PL, Horwitz S, Moskowitz A, Querfeld C. Primary cutaneous T-cell lymphoma (mycosis fungoides and Sézary syndrome): part II. Prognosis, management, and future directions. J Am Acad Dermatol. 2014;70:223.e1-17.

7. Carter J, Zug KA. Phototherapy for cutaneous T-cell lymphoma: online survey and literature review. J Am Acad Dermatol. 2009;60:39-50.

8. Ponte P, Serrão V, Apetato M. Efficacy of narrowband UVB vs. PUVA in patients with early-stage mycosis fungoides. J Eur Acad Dermatol Venereol. 2010;24:716-21.

9. Ahmad K, Rogers S, McNicholas PD, Collins P. Narrowband UVB and PUVA in the treatment of mycosis fungoides: a retrospective study. Acta Derm Venereol. 2007:87:413-7.

10. Boztepe G, Sahin S, Ayhan M, Erkin G, Kilemen F. Narrowband ultraviolet B phototherapy to clear and maintain clearance in patients with mycosis fungoides. J Am Acad Dermatol. 2005;53:242-6.

11. Yones SS, Palmer RA, Garibaldinos TT, Hawk JL. Randomized double-blind trial of the treatment of chronic plaque psoriasis: efficacy of psoralen-UV-A therapy vs narrowband UV-B therapy. Arch Dermatol. 2006;142:836-42.

12. Markham T, Rogers S, Collins P. Narrowband UV-B (TL-01) phototherapy vs oral 8-methoxypsoralen psoralen-UV-A for the treatment of chronic plaque psoriasis. Arch Dermatol. 2003:139:325-8.

13. Farage MA, Miller KW, Berardesca E, Maibach HI. Clinical implications of aging skin: cutaneous disorders in the elderly. Am J Clin Dermatol. 2009;10:73-86.
14. Seckin D, Demircay Z, Akin O. Generalized pruritus treated with narrowband UVB. Int J Dermatol. 2007;46:367-70.

15. Rivard J, Lim HW. Ultraviolet phototherapy for pruritus. Dermatol Ther 2005;18:344-54.

16. Samson Yashar S, Gielczyk R, Scherschun L, Lim HW. Narrow-band ultraviolet B treatment for vitiligo, pruritus, and inflammatory dermatoses.

17. Photodermatol Photoimmunol Photomed. 2003;19:164-8.

18. Gamil H, Nassar A, Saadawi A, El-Qashishi K, Ahmed F. Narrow-band ultraviolet B phototherapy in lichen planus. J Eur Acad Dermatol Venereol. 2009;23:589-90.

19. Pavlotsky F, Nathansohn N, Kriger G, Shpiro D, Trau H. Ultraviolet-B treatment for cutaneous lichen planus: our experience with 50 patients. Photodermatol Photoimmunol Photomed. 2008;24:83-6.

20. Saricaoğlu H, Karadogan SK, Başkan EB, Tunali S. Narrowband UVB therapy in the treatment of lichen planus. Photodermatol Photoimmunol Photomed. 2003;19:265-7.

21. Martin JA, Laube S, Edwards C, Gambles B, Anstey AV. Rate of acute adverse events for narrow-band UVB and Psoralen-UVA phototherapy. Photodermatol Photoimmunol Photomed. 2007;23:68-72.

How to cite this article: Bulur I, Kaya Erdogan H, Aksu AE, Karapınar T, Saracoglu ZN. The efficacy and safety of phototherapy in geriatric patients: a retrospective study. An Bras Dermatol. 2018;93(1):33-8. 